\title{
Skin Autofluorescence Profile in Tunisian Subjects with and Without Metabolic Disorders
}

\section{Ines Khochtali}

Fattouma Bourguiba University Hospital

\section{Wahid Bouida}

Fattouma Bourguiba University Hospital

Hamdi Boubaker

Fattouma Bourguiba University Hospital

\section{Semir Nouira}

Fattouma Bourguiba University Hospital

Mohsen Kerkeni ( $\sim$ mohsen.kerkeni@yahoo.fr)

Higher Institute of Biotechnology

\section{Research Article}

Keywords: Skin autofluorescence, advanced glycation end products, metabolic disorders

Posted Date: November 2nd, 2021

DOI: https://doi.org/10.21203/rs.3.rs-1028612/v1

License: (c) (1) This work is licensed under a Creative Commons Attribution 4.0 International License. Read Full License 


\section{Abstract}

Background: The AGE Reader, as a clinical tool for non invasive assessment, measures the accumulation of advanced glycation end products (AGEs) in skin tissues shown as skin autofluorescence (SAF). AGEs Accumulation has been implicated in several diseases. There is no data about SAF profile in Tunisian population, this study aimed to assess firstly SAF profile in subjects with and without metabolic disorders and secondly to examine the association between SAF and various clinical parameters.

Methods: In a cross-sectional study, we included 220 participants between 19 and 86 years of age who were subdivided in two groups: a healthy group $(n=93)$ and patients group with metabolic disorders $(n=127)$ contains three subgroups as following: diabetic patients $(n=32)$, hypertensive patients $(n=54)$ and patients with both diabetes and hypertension $(n=41)$. Skin AGEs accumulation was measured by AGE Reader and clinical data was obtained.

Results: SAF was significantly higher in patients group compared to healthy group [2.40 AU (2.10-2.60) vs. $2.00 \mathrm{AU}(1.70-2.10)$ respectively; $P<0.001]$. Diabetic patients and hypertensive patients showed an increased level of SAF. The highest of SAF was observed in patient with both coexistence of diabetes, hypertension and dyslipidemia. SAF was associated with age, gender, BMI, duration of diabetes, HbA1c, triglyceride and obesity. Multivariate analysis showed that age and duration of diabetes were independent determinant of SAF. The ROC analysis indicated that an SAF > 2.25 AU was optimal cut-off point to predict the presence of metabolic disorders $(P<0.001)$.

Conclusion: SAF was increased in patients with diabetes and/or with hypertension and dyslipidemia. AGE Reader device is a rapid and helpful tool in clinical practice for evaluating and screening metabolic disorders in undiagnosed subjects.

\section{Introduction}

Diabetes Mellitus and dyslipidemia as known as metabolic disorders explained by glucose and lipid metabolic abnormalities. Hypertension is related to impaired metabolic homeostasis and can be regarded as a metabolic disorder [1,2]. The numbers of subjects suffering for metabolic disorders are constantly increasing in Tunisia [3-5]. Type 2 diabetes, a silent disease, induces long-term vascular complications and is diagnosed too late [6,7]. Diabetic patients which are under treatment do not prevent to develop microvascular complications (retinopathy, nephropathy and neuropathy) and macrovascular complications (cerebrovascular disease, cardiovascular disease and peripheral vascular disease) which that contribute to the increased risk of morbidity and mortality [8]. It is well known that hyperglycemia induces glycation and oxidative pathways directly or indirectly on proteins, lipids and nucleic acids $[9,10]$. Glucose and glycated hemoglobin (HbA1c) levels are the gold reference biomarkers for detecting diabetes but these parameters are usually fluctuated in the blood and do not reflect the profile in tissues for diabetic patients with or without vascular complications. Currently, several studies have shown the role of 
advanced glycation end products (AGEs) accumulation in tissues as biomarker for early detection, screening and diagnosis of many diseases $[11,12]$.

AGEs are a complex and heterogeneous group of non-fluorescent and fluorescent compounds becomes irreversibly cross-linked adducts which bind to amino acids (lysine/arginine residues) of proteins, and accumulate on proteins a slow turnover [13]. AGEs are formed by a non enzymatic glycation and oxidation during aging and are much rise in hyperglycemia conditions. Many different AGEs have been identified, such as, pentosidine, crossline, methyl-glyoxal-lysine dimmer (MOLD), glyoxal-lysine dimmer (GOLD) as fluorescent AGEs, and N-carboxymethyl-lysine (CML), methyl-imidazolone, argpyrimidine, glucosepane, pyrriline as a non-fluorescent AGEs $[9,10]$.

We have shown previously that plasma AGEs has been implicated in diabetes with and without vascular complications in Tunisian patients [14-16]. previous studies have shown that skin AGEs accumulation was measured non invasively by AGE Reader device as a simple tool to identify those at risk for diabetes and those at risk for diabetes complications $[17,18]$. There is no study about skin AGEs accumulation in Tunisian subjects with and without metabolic disorders. This cross-sectional study, we have measured SAF in healthy subjects and in patients, and we have examined the relationship between the SAF and various clinical parameters.

\section{Methods}

\section{Study population}

For the current study, we have included 220 participants from Monastir region (Tunisia) who provided written informed consent. The study protocol was approved by the medical ethical review committee of the hospital Fattouma Bourguiba at Monastir. All participants are subdivided in two groups: one group for healthy control subjects between 19 and 73 years old $(n=93)$, the second group for patients between 36 and 86 years old $(n=127)$ without history for cardiovascular or kidney diseases and contains three subgroups as following: diabetic patients $(n=32)$, hypertensive patients $(n=54)$ and patients with both diabetes and hypertension ( $n=41$ ). Diabetes is defined as fasting blood glucose $\geq 7.0 \mathrm{mmol} / \mathrm{L}$ or $\mathrm{HbA} 1 \mathrm{c} \geq 6.5 \%$ and/or use of anti-diabetes medication Hypertension is defined as systolic blood pressure $\geq 130 \mathrm{mmHg}$ and/or diastolic blood pressure $\geq 85 \mathrm{mmHg}$ and/or use of anti-hypertensive medication. Dyslipidemia is defined as hypercholesterolemia and/or hypertriglyceridemia and/or use of lipid lowering medication. Obesity is defined as $\mathrm{BMI} \geq 30 \mathrm{~kg} / \mathrm{m}^{2}$. The three subgroup of patient are with or without dyslipidemia.

\section{Clinical data collection}

For each subject we have collected the following data: Age, sex, body mass index (BMI), duration of diabetes, diabetes, hypertension and dyslipidemia.

\section{Skin autofluorescence measurement}


For all participants, SAF was measured by AGE Reader device (Diagnotics, Groningen, Netherlands) and the method has been described previously $[19,20]$. The device, a non-invasive tool, evaluates AGEs in skin using the principle that several AGEs emit autofluorescence when excited by UV light. Excitation light source which wavelength is between 300 and $420 \mathrm{~nm}$ and the peak intensity is at $370 \mathrm{~nm}$. This light source is projected approximately onto $4 \mathrm{~cm}^{2}$ on the volar side of the forearm skin surface, and the intensity of any light $(420-600 \mathrm{~nm})$ emitted is measured with an internal spectrometer. SAF was expressed in arbitrary units $(A U)$ and was calculated from the mean value of the emitted light intensity divided by the excitation light intensity and multiplying by 100 . The patients with Fitzpatrick phototypes $\mathrm{V}$ and $\mathrm{VI}$ were not included due their skin pigmentation, which had ultraviolet reflectance of < $6 \%$. The reproducibility of SAF is indicated by a mean coefficient of variance $\sim 5 \%$ [19]. For every participant, SAF was measured three times on normal skin without scars, tattoos, or other skin abnormalities.

\section{Statistical analysis}

Data are shown as mean \pm standard deviation (SD) or median and interquartile range in case of non-normally distributed data. Between groups comparisons were performed using the Student's $t$-test or Mann-Whitney test, and the correlation coefficient was estimated using the Person or Spearman rankorder correlation analysis. Multivariate analysis was performed using multiple regression analyses. The receiver-operating characteristic curves were constructed to determine the optimal SAF cut-off levels for prediction of metabolic disorders. A $P$-value $<0.05$ was considered statistically significant. All of statistical analyses were performed using SPSS-17.0 statistical software (IBM, USA).

\section{Results}

\section{Characteristics of the study population}

The demographic and clinical characteristics of the study population are presented in Table 1. A significant increased was shown for age, $\mathrm{BMl}$, glucose, $\mathrm{HbA} 1 \mathrm{c}$, total cholesterol and triglyceride levels in patients compared to healthy subjects. All patients showed a high proportion of hypertension, diabetes and dyslipidemia $(74.8 \%, 57.7 \%$, and $32.2 \%$ respectively). The median SAF value was increased in patients compared to healthy subjects, as shown in Figure $1(P<0.001)$. SAF levels in healthy controls aged between 19 and 73 years old has a mean value at $1.90 \pm 0.29 \mathrm{AU}$. Compared to patients group the mean age of healthy controls was significantly lower. To matched age between the two groups, SAF levels in healthy group $(n=67)$ shown that the mean value was at $2.00 \pm 0.22 \mathrm{AU}$. This SAF value still significant lower compared to patients group $(P<0.001)$.

\section{SAF levels in patient subgroups}

The SAF levels in patient subgroups are presented in Table 2. For each patient subgroup SAF levels were markedly increased compared to healthy subjects. Patients with both DM and HTA showed higher age and a significant increased SAF levels compared to patients with HTA $(P=0.047)$. 


\section{Correlation of SAF levels with other variables}

The relationship between SAF levels and other variables in study population was shown in table 3. For healthy subjects, univariate linear regression analysis showed a significant relationship between $\mathrm{SAF}$, age, BMI and obesity. Multivariate analysis showed that age was independent determinant of SAF ( $b$ $=0.552, P<0.001)$. For patients group, univariate analysis showed a significant relationship between $\mathrm{SAF}$, age, gender, duration of diabetes, $\mathrm{HbA} 1 \mathrm{c}$ and triglyceride levels. Multivariate analysis showed that age and duration of diabetes were independent determinant of SAF $(b=0.270, P=0.008 ; b=0.515, P<$ 0.001 respectively). For all participants a significant correlation was shown between SAF and HbA1c levels $(r=0.361, P<0.001)$ (Figure 2).

\section{Receiver operating characteristic curve}

To predict the presence of metabolic disorders based on SAF, receiver operating characteristic curve was used and shown in figure 3. The maximum Youden index indicated that an SAF > 2.25AU was optimal cut-off point to predict the presence of metabolic disorders. Area under the curve (AUC) $=0.844$ (95\%Cl: $0.795-0.894), P<0.001$ with sensitivity of $63 \%$ and specificity of $92 \%$.

\section{Discussion}

This is the first study showing that the SAF profile in Tunisian population. The SAF values were not the same in different ethnicities, as described by Mook-Kanamori et al. Authors showed that Arabs and Filipinos had a significant higher SAF than the South Asian population. In Arab population (200 subjects from 15 Arabs countries), SAF levels were 2.09 (1.47-3.31) in 115 healthy subjects and 2.64 (1.67-4.02) in 85 patients with DM [21]. Other study done by Ahmad et al. showed that the effects of seven deferent ethnicities: Arab, Central-East African, Eastern Mediterranean, European, North African, South Asian and Southeast Asian on SAF in Arabian cohort. They have shown Highest SAF values were observed for the North African population, followed by East Mediterranean, Arab, South Asian and European populations [22]. For our study, as Arab population, resident in Tunisia country on North of Africa, we found in Tunisian healthy subjects $(n=93)$ the SAF levels were 2.00 (1.70-2.10) and in Tunisian patients $(n=127)$ the SAF levels were 2.40 (2.10-2.60). Difference between the above Arab studies may be explained by two major raisons: the lifestyle and habitual dietary adapted in Tunisian population. For gender, our study showed no significant difference in healthy group [Men: 1.80 (1.60-2.10); Women: 2.00 (1.70-2.12)]. However, a significant difference was shown in patients group [Men: 2.30 (2.00-2.50); Women: 2.40 (2.202.70); $P=0.014$ ]. Our results showed only a significant increased SAF values in women patients. For other Arab studies, authors showed that gender affect SAF levels on both groups of healthy and patients $[21,22]$. Cigarette smoking can also affect the SAF levels and previous studies showed that cigarette smokers had higher levels than nonsmokers $[20,23]$. Due to small size for our smokers participants data were not shown in this study and patient participants have stopped smoking for a longtime.

Tunisian subjects with metabolic disorders showed an increased SAF levels compared to healthy subjects. Diabetic patients showed the same SAF levels with hypertensive patients, however, patients with 
both metabolic disorders showed an increased SAF levels. Our finding about elevated levels of SAF in diabetes and in hypertension is line with results of several previous studies [12, 24]. In subjects with diabetes, the formation of AGEs is accelerated due to hyperglycemia. Glucose plays an essential role in the formation of AGEs as protein amino groups and lipids are non-enzymatically glycated to form stable structures on long lived tissues [25]. AGEs formation also produced by glucose autoxidation, lipid peroxidation and by the reactive carbonyl compounds [10]. Elevated blood pressure may well be a consequence of increased AGEs accumulation. Several AGEs are able to form crosslinks within collagen in the vascular wall, which may result in impaired vascular elasticity and increased arterial stiffness, causing blood pressure to rise [26]. Also several metabolites released from metabolic dysfunction were associated with elevated blood pressure as described recently [2]. These observations may us to suggest that AGEs could influence directly or indirectly blood pressure via modulation of metabolic processes.

The prevalence of diabetes and hypertension in Tunisia is growing rapidly and it is so important to use non invasive technologies in clinical practice to detect early diseases and monitor patient's health over time. The AGE Reader is a non invasive device that measures tissue accumulation of AGEs provides also an immediate vascular risk prediction. Several studies have already shown the usefulness of SAF as a new marker in predicting diabetes and its complications [17-19].

In the present study we found significant associations between SAF levels and other additional factors such as age, gender, BMI, duration of diabetes, $\mathrm{HbA1c}$ and obesity. Our finding is a line with several studies that they have shown the same results [27-31]. Moreover, we found that age and diabetes duration were independent risk factors that were associated with SAF. The screening value of SAF for the presence of metabolic disorders was assessed by ROC analysis and revealed that the optimal cut-off value was $2.25 \mathrm{AU}$ which suggests that it could be used as a prognostic indicator.

Some limitations of this study should be noted. The number of healthy subjects and patients seems small and the value of SAF in predicting for metabolic disorders could not confirmed in this crosssectional study, which requires further follow-up.

In conclusion, this study indicates that SAF was associated with the presence of metabolic disorders. Non invasive measurement of SAF is a good surrogate marker for evaluating metabolic disorders. Further investigations in a large number of prospective studies are required to validate the results in this study.

\section{Abbreviations}

AGEs: Advanced glycation end products; SAF: Skin autofluorescence; BMI: Body mass index; DM: Diabetes Mellitus; HTA: Hypertension.

\section{Declarations}

The study protocol is in accordance with ethical guidelines of declaration of Helsinki and has approved by the ethics committees at the Hospital of Fattouma Bourguiba at Monastir-Tunisia. All participants 
signed the informed consent in writing prior to inclusion in the study.

\section{Acknowledgments}

The authors would like to thank Professor Bruce H. R. Wolffenbutel from Department of Endocrinology, University of Groningen, University Medical Center Groningen, the Netherlands for sending the AGE Reader device to our clinical research.

\section{Authors' contributions}

MK and SN conceived the idea, edited and finalized the manuscript. IK and MK: Performed the study design, skin autofluorescence measurement, data analysis, data interpretation, and writing. WB and HB: Helped in the recruitment of patients and clinical fulfillment. All authors read and approved the final manuscript.

\section{Funding}

Not applicable

\section{Availability of data and materials}

The datasets used and/or analysed during the current study are available from the corresponding author on reasonable request.

\section{Consent for publication}

Not applicable

\section{Competing interests}

The authors declare that they have no conflict of interest to disclose.

\section{References}

1. Tanaka $\mathrm{M}$, Itoh $\mathrm{H}$. Hypertension as a metabolic disorder and the novel role of the gut. Current hypertension reports. 2019;21:63.

2. Chakraborty S, Mandal J, Yang T, Cheng X, Yeo JY et al. Metabolites and hypertension: Insights into hypertension as a metabolic disorder. Hypertension. 2020;75:1386-1396.

3. Ben Romdhane H, Ben Ali S, Aissi W, Traissac P, Aounallah-Skhiri H, Bougatef S et al. Prevalence of diabetes in Northern African countries: the case of Tunisia. BMC Public Health. 2014;14:86.

4. Maoui A, Bouzid K, Ben Abdelaziz A, Ben abdelaziz A. Epidemiology of type 2 diabetes in the geater Maghreb. Exemple of Tunisia. Systematic review of the literature. La Tunisie Medicale. 2019; Vol 97. 
5. Romdhane HB, Ali SB, Skhiri H, Traissac P, Bougatef S, Maire B et al. Hypertension among Tunisian adults: results of the TAHINA project. Hypertens Res. 2012;35(3):341-347.

6. Kahloun R, Jelliti B, Zaouali S, Attia S, Ben Yahia S, Resnikoff S, Khairallah M. Prevalence and causes of visual impairment in diabetic patients in Tunisia, North Africa. Eye. 2014;28:986-991.

7. Kechida M. Cardio-metabolic risk factors in Tunisia: state of the art. Intern Emerg Med. 2020;15(4):537-542.

8. Elisa Dal Canto, Antonio Ceriello, Lars Rydén, Marc Ferrini, Tina B Hansen, Oliver Schnell, Eberhard Standl, Joline WJ Beulens. Diabetes as a cardiovascular risk factor: An overview of global trends of macro and micro vascular complications. Eur J Prev Cardiol. 2019;26(2suppl):25-32.

9. Brownlee M. Lilly lecture 1993. Glycation and diabetes complications. Diabetes. 1994;43:836-841.

10. Baynes JW, Thorpe SR. Glycoxidation and lipoxidation in atherogenesis. Free Radic Biol Med. 2000;28:1708-1716.

11. Van Waateringe RP, Fokkens B, Slagter SN, van der Klauw MM, van Vliet-Ostaptchouk JV et al. Skin autofluorescence predicts incident type 2 diabetes, cardiovascular disease and mortality in general population. Diabetologia. 2019;62:269-280.

12. Van Waateringe RP, Slagter $S N$, van Beek AP, van der Klauw MM, van Vliet-Ostaptchouk JV, Graaff R, et al. Skin autofluorescence, a non-invasive biomarker for advanced glycation end products, is associated with the metabolic syndrome and its individual compents. Diabetol Metab Syndr. 2017;9:42.

13. Schmitt A, Schmitt J, Münch G, Gasic-Milencovic J. Characterization of advanced glycation end products for biochemical studies: side chain modifications and fluorescence characteristics. Anal Biochem. 2005; 338:201-215.

14. Kerkeni M, Saïdi A, Bouzidi H, Ben Yahya S, Hammami M. Elevated serum levels of AGEs, sRAGE, and pentosidine in Tunisian patients with severity of diabetic retinopathy. Microvasc Res. 2012;84(3):37883.

15. Kerkeni M, Saïdi A, Bouzidi H, Ben Yahya S, Hammami M. Pentosidine as a biomarker for microvascular complications in type 2 diabetic patients. Diab Vasc Dis Res. 2013;10(3):239-45.

16. Kerkeni M, Weiss IS, Jaisson S, Dandana A, Addad F, Gillery P, Hammami M. Increased serum concentrations of pentosidine are related to presence and severity of coronary artery disease. Thromb Res. 2014;134(3):633-8.

17. Fokkens BT, Smit AJ. Skin fluorescence as a clinical tool for non-invasive assessment of advanced glycation and long-term complications of diabetes. Glycoconj J. 2016;33:527-535.

18. Fokkens BT, van Waateringe RP, Mulder DJ, Wolffenbuttel BHR, Smit AJ. Skin autofluorescence improves the Finnish Diabetes Score in the detection of diabetes in a large population based cohort: The LifeLines Cohort Study. Diabetes \& Metabolism. 2018;44:424-430.

19. Meerwaldt R, Graaff R, Oomen PH, Links TP, Jager JJ, Alderson NL, et al. Simple non-invasive assessment of advanced glycation endproduct accumulation. Diabetologia. 2004;47:1324-1330. 
20. Koetsier M, Lutgers HL, de Jonge C, Links TP, Smit AJ, Graaff R. Reference values of skin autofluorescence. Diabetes Technol Ther. 2010;12:399-403.

21. Mook-Kanamori MJ, El-Din Selim MM, Takiddin AH, Al-Homsi H, Al-Mahmoud KAS, Al-Obaidli A et al. Ethnic and gender differences in advanced glycation end products measured by skin autofluorescence. Dermato-Endocrinology. 2013;5:325-330.

22. Ahmad MS, Kimhofer T, Ahmad S, AIAma MN, Mosli HH, Hindawi SI, Mook-Kanamori DO et al. Ethnicity and skin autofluorescence based risk engines for cardiovascular disease and diabetes mellitus. PLoS ONE.2017;12(9):e0185175.

23. Van Waateringe RP, Mook-Kanamori MJ, Slagter SN, van der Klauw MM, van Vliet-Ostaptchouk JV, Graaff $\mathrm{R}$, et al. The association between various smoking behaviors, cotinine biomarkers and skin autofluorescence. PLoS ONE. 2017;12(6):e0179330.

24. Botros N, Sluik D, van Waateringe RP, de Vries JH, Geelen A, Feskens EJ. Advanced glycation end products (AGEs) and associations with cardio-metabolic, lifestyle and dietary factors in a general population: the NQplus study. Diabetes Metab Res Rev. 2017;17:259.

25. Brownlee M. Advanced protein glyocsylation in diabetes and aging. Annu Rev Med. 1995;46:223-34.

26. Aronson D. Cross-linking of glycated collagen in the pathogenesis of arterial and myocardial stiffening of aging and diabetes. J Hypertens. 2003; 21:3-12.

27. Noordzij MJ, Mulder DJ, Oomen PHN, Brouwer T, Jager J, Castro Cabezas M, et al. Skin autofluorescence and risk of micro- and macrovascular complications in patients with diabetes mellitus a multi-center study. Diabt Med. 2012;29:1556-61.

28. Lutgers HL, Graaff R, Links TP, Ubink-VeltmaatLJ, Bilo HJ, Gans RO, et al. Skin autofluorescence as a noninvasive marker of vascular damage in patients with type 2 diabetes. Diabetes care. 2006;29:2654-9.

29. den Engelsen C, van den Donk M, Gorter KJ, Salomé PL, Rutten GE. Advanced glycation end products measured by skin autofluorescence in a population with central obesity. Dermato Endocrinol. 2012;4:33-8.

30. Noordzij MJ, Lefrandt JD, Graaff R, Smit AJ. Skin autofluorescence and glycemic variability. Diabetes Technol Ther. 2010;12:581-5.

31. Kellow NJ, Coughlan MT, Reid CM. Association between habitual dietary and lifestyle behaviours and skin autofluorescence (SAF), a marker of tissue accumulation of advanced glycation endproducts (AGEs), in healthy adults. Eur J Nutr. 2018;57:2209-2216.

\section{Tables}

\section{Table 1}

\section{Characteristics of the study population}




Characteristics $\quad$ Healthy subjects $(\mathrm{n}=93) \quad$ Patients $(\mathrm{n}=127) \quad P$

Age (years)

$43 \pm 14$

$57 \pm 9$

$<0.001$

Male gender, n (\%)

$31(33.3 \%)$

$53(41.7)$

0.305

BMI $\left(\mathrm{kg} / \mathrm{m}^{2}\right)$

$27.91(24.65-30.72)$

$30.21(27.68-32.95)$

$<0.001$

Diabetes, n (\%)

$-$

$73(57.5 \%)$

Duration of diabetes (years)

$-$

$8(3-14)$

Dyslipidemia, n (\%)

$41(32.3 \%)$

Hypertension, n (\%)

$95(74.8 \%)$

Blood glucose (mmol/L)

$5.60(5.40-5.80)$

$8.60(8.40-8.90)$

$<0.001$

Hemoglobin A1c (\%)

$5.40(5.20-5.60)$

$6.80(5.77-8.00)$

$<0.001$

Total cholesterol (mmol/L)

$4.70(4.50-4.90)$

$5.30(4.90-5.60)$

$<0.001$

Triglyceride (mmol/L)

$1.20(0.80-1.40)$

$1.90(1.50-2.10)$

$<0.001$

Skin autofluorescence (AU)

$2.00(1.70-2.10)$

$2.40(2.10-2.60)$

$<0.001$

Data are shown as the median (interquartile range), mean \pm SD or number (percentage).

BMI: body mass index

\section{Table 2}

Characteristics of patient subgroups

Characteristic

Patients $(n=127)$

$$
\mathrm{DM}(\mathrm{n}=32)
$$

HTA $(n=54)$

DM \& HTA $(n=41)$

Age (years)

$$
55 \pm 8
$$

$57 \pm 10$

$60 \pm 8^{a}$ 

BMI $\left(\mathrm{kg} / \mathrm{m}^{2}\right)$
$29.78 \pm 5.14$
$31.18 \pm 5.98$
$31.11 \pm 3.74$

Dyslipidemia, n (\%)

$8(25 \%)$

$21(38.88 \%)$

$12(29.26 \%)$

SAF (AU)

$2.40(2.15-2.65)$

$2.30(2.00-2.50)$

$2.40(2.20-2.80)^{b}$

Data are shown as the median (interquartile range), mean \pm SD or number (percentage).

a $P=0.023$ compared to patients with DM

b $P=0.047$ compared to patients with HTA

BMI: body mass index

\section{Table 3}

Relative risk factors of SAF in study population

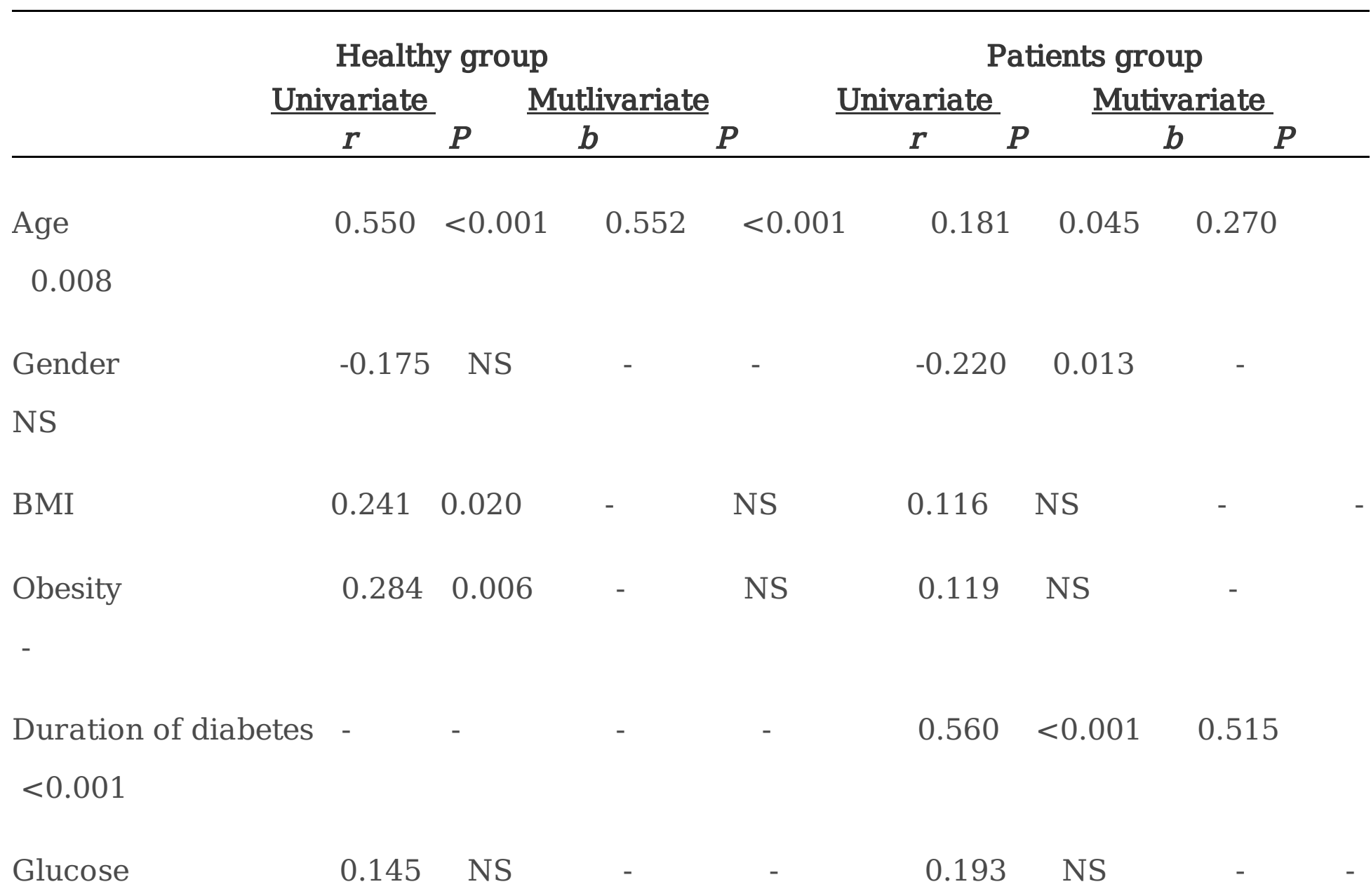


- -

0.240

0.011

NS

Cholesterol

$0.163 \quad$ NS

- -

$0.188 \quad$ NS

Triglyceride

$0.197 \quad$ NS

0.236

0.010

Linear regression analysis was performed to determine predictors of SAF. The significant variables in the univariate analysis were selected for the multivariate regression analysis. NS: not significant.

Figures

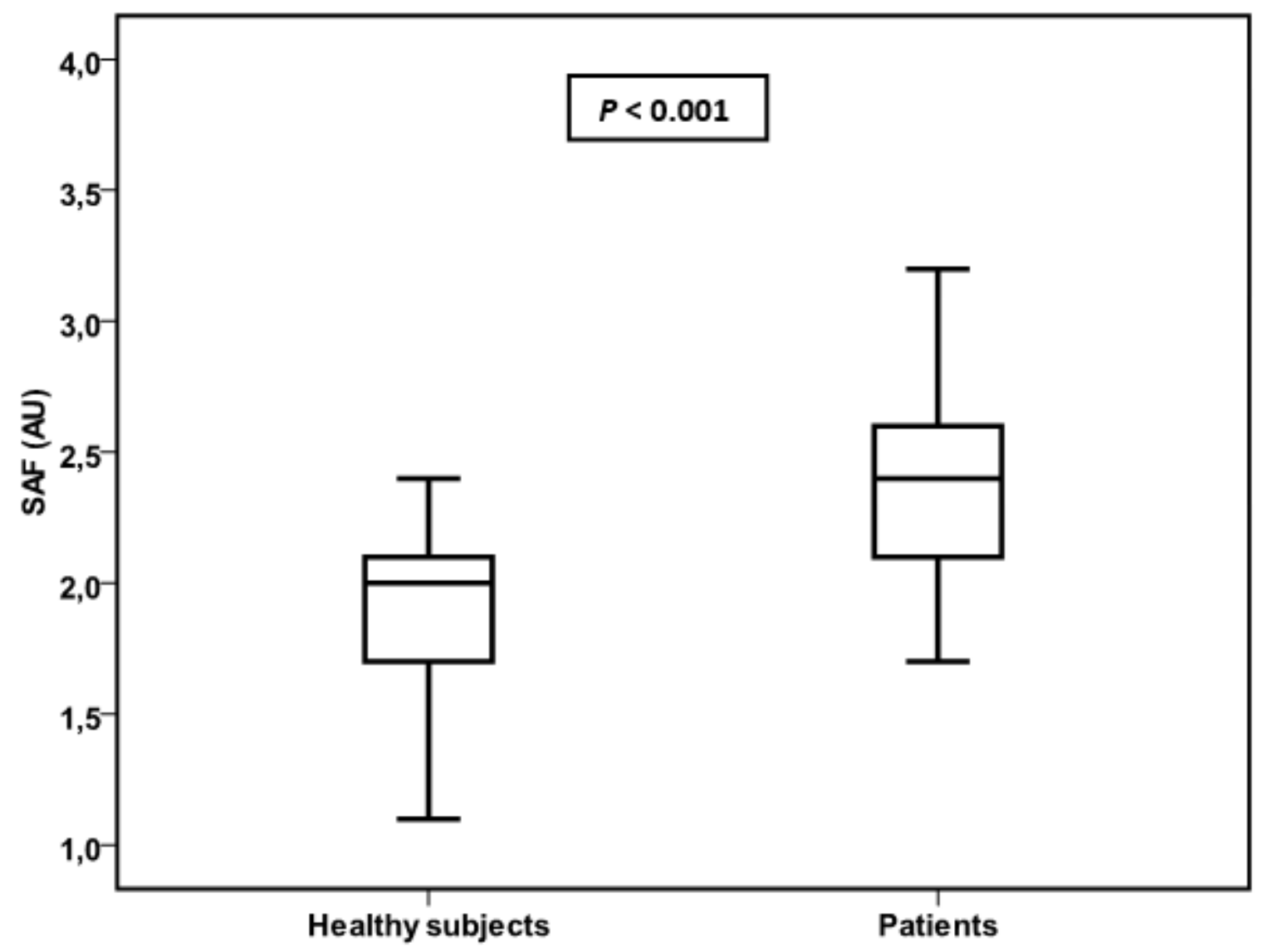

Figure 1 
SAF levels in study population.

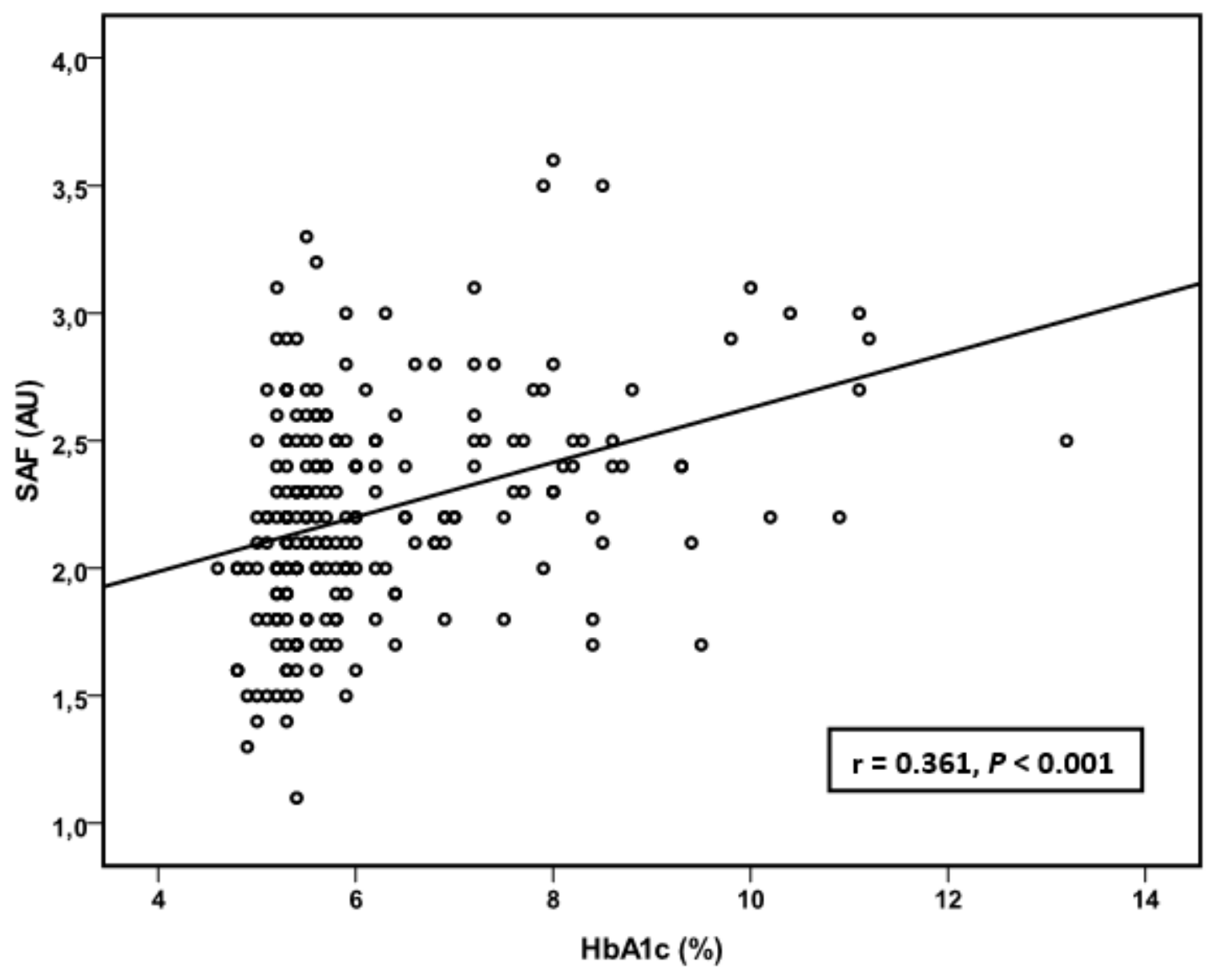

Figure 2

Correlation between SAF and HbA1c in study population ( $n=220)$. 


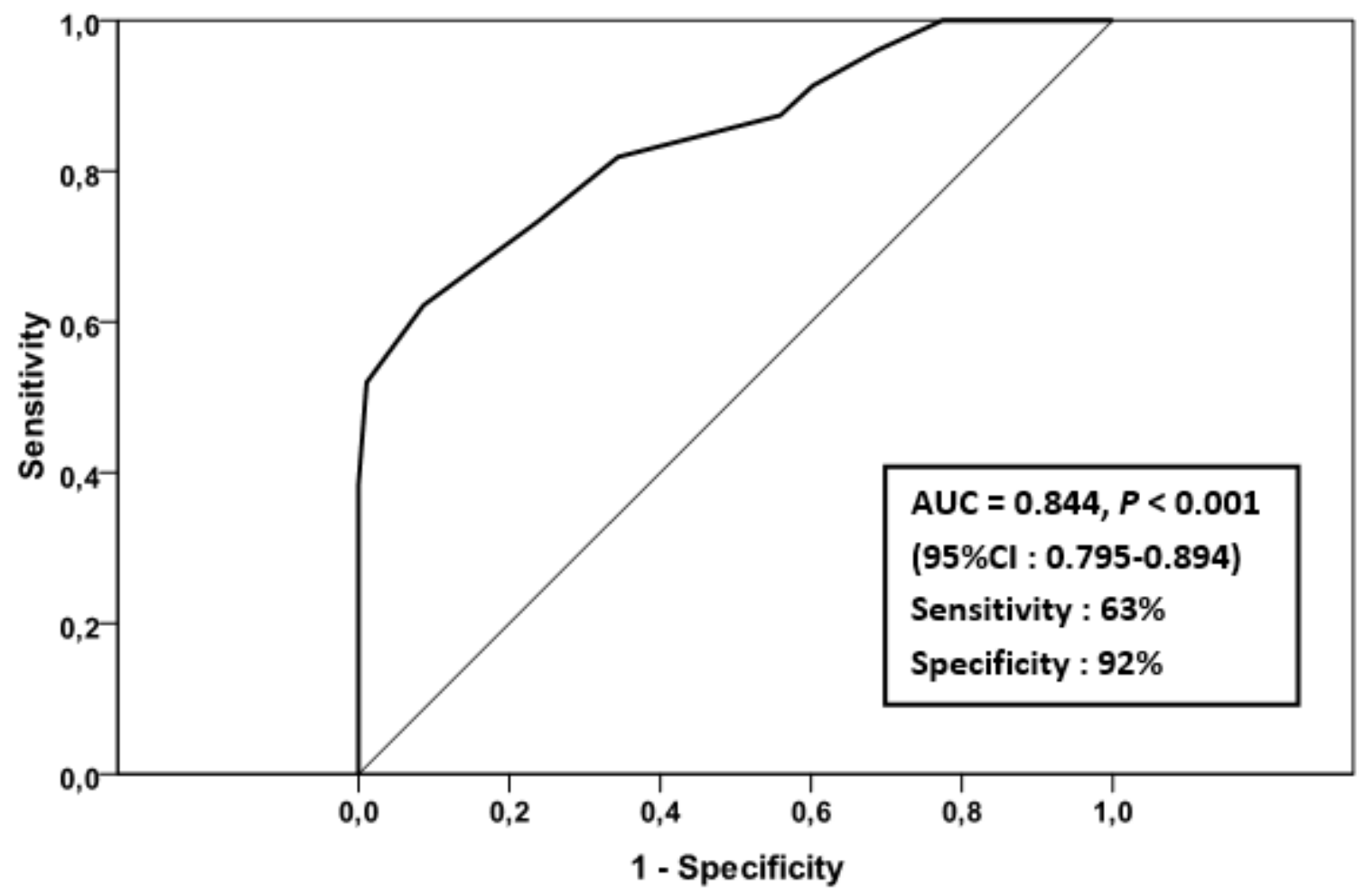

Figure 3

The receiver operating characteristic curve analysis for prediction the presence of metabolic disorders (DM and/or HTA and dyslipidemia) based on SAF. Maximum Youden index indicated that a SAF $>2.25$ AU was optimal cut-off point to predict metabolic disorders. AUC: area under the curve 